\title{
COMPETITIVE INTERACTIONS IN DROSOPHILA MELANOGASTER III. TRIOCULTURES
}

\author{
P. D. S. CALIGARI* AND KENNETH MATHER ${ }^{\dagger}$ \\ * Scottish Crop Research Institute (Pentlandfield), Roslin, Midlothian EH25 9RF; \\ tDepartment of Genetics, University of Birmingham, Birmingham B15 $2 \pi$
}

Received 9.ix.83

\section{SUMMARY}

\begin{abstract}
Three inbred lines from the Texas population, (X1, X5 and X15), whose behaviour in respect of character $p_{a}$ (the proportion of eggs successfully developing into adults, transformed into angles) in monoculture and duoculture has been analysed by Mather and Caligari (1983), were also raised in trioculture in the same experiment. The triocultures were set up on the same essential basis as duocultures, the various numbers of eggs of one line (the unique genotype) being complemented by eggs taken in equal numbers from the two other lines (the paired genotypes). Three different types of trioculture are possible with this arrangement.

Taking the unique genotype as indicator, the competitive pressure exerted on it by the paired genotypes jointly, can be compared with the pressures exerted by the two genotypes separately in duoculture. Although the joint pressure did not depart significantly from the simple mean of the two individual genotypes, it fitted better with the mean weighted according to the relative proportions of the two genotypes among the individuals from the triocultures and from their duocultures. This suggests that the effects of the competition between the paired genotypes themselves are reflected in their joint pressure on the unique genotype, and that competitive pressures exerted by different genotypes are simply additive when account is taken of their effective relative numbers in the culture.

Because of a deficiency in the design of the experiment the pressure exerted by the unique genotype on the paired components of the triocultures cannot be analysed in the same way. However, comparison of the values of $p_{a}$ in the paired genotypes in trioculture with those in the duoculture to which they contributed equal numbers of eggs, agreed well with a simple additive relation in the competitive pressures exerted jointly by different genotypes.
\end{abstract}

\section{INTRODUCTION}

In the earlier papers of this series (Caligari, 1980; Mather and Caligari, 1981) we have considered the principles upon which could be based experiments seeking to measure and compare the intra-genotypic competition between individuals of like genotype and the inter-genotypic competition between unlike genotypes, and we have developed a methodology of experiment and analysis for achieving these ends using Drosophila melanogaster as the experimental material. In later papers this approach has been extended to competition within and between genotypes of the perennial rye-grass Lolium perenne (Mather, Hill and Caligari, 1982), and using data from both rye-grass and a wider range of Drosophila experiments it has been shown how the two components of competition, viz., the competitive pressure (a) exerted by an individual on all its fellow individuals of the population and the response $(r)$ of the individual to the sum of the competitive pressures, exerted by its fellows, can be separately evaluated and their relations investigated (Mather and Caligari, 1983). 
In all the experiments so far considered, use has been made only of series of monocultures, where cultures of varying density are raised using individuals all of like genotype, and duocultures, where two genotypes are mixed in varying proportion. Clearly, however, if the approach is to be of value in considering natural populations or even many situations that arise in agriculture, it must be extended to cover mixtures of more than two genotypes, to permit investigation of the way in which competitive pressures from different genotypes combine and the way in which this combination is affected by the competition between aggressor genotypes in exerting their effects on individuals of yet a different genotype. The experiment whose results will now be considered involved mixtures of three genotypes, which is the simplest case where such questions arise.

\section{THE EXPERIMENT}

The three inbred lines (X1, X5 and X15) used in the experiment were derived from the Texas cage population which has been maintained in Birmingham for many years. Since all three lines were wild-type, the marker genes $w^{a}$ and $y^{2}$ were introduced into X5 and X15 respectively to facilitate classification of the individuals emerging in mixed cultures. The method of introduction is described briefly by Mather and Caligari (1983), who also report evidence from other experiments that $y^{2}$ does not of itself affect the competitive abilities of individuals displaying its effects. No similar test has been carried out for $w^{a}$, but equally no suggestion of any such effect has been observed in the present experiment.

Three series of monocultures were raised, one for each of the three genotypes, and also three series of duocultures covering all the combinations of the genotypes taken two at a time. The reference density of eggs per culture was 120 , and $x$ took the values $0,-30,-60$ and -90 , so giving densities of $120,90,60$ and 30 eggs of the sole genotype in monoculture, and 90,60 and 30 of the indicator genotype in duocultures, where of course either genotype can be taken as the indicator. $45 \mathrm{mgm}$ of yeast were added to each culture vial. The character recorded was $p_{a}$, the proportion of eggs of the indicator genotype that developed successfully into adult flies, this proportion being transformed into angles to equalise the basic sampling variances. The experiment was carried out in duplicate, the differences between the duplicates providing the estimate of error variation, in the calculation of which a correction was made for the overall difference between the duplicate series.

This part of the experiment is a self-contained example of our customary design for the investigation of competitive interactions between pairs of genotypes (see Mather and Caligari, 1981) and can be analysed as such. The results of the analysis have been given in section 3(ii)c of Mather and Caligari (1983). The results from the triocultures which formed the remainder of the experiment have not, however, been reported earlier.

Three series of triocultures were raised alongside the mono- and duocultures. In each series one of the three genotypes was used at densities of $120+x$, where $x$ was again $-30,-60$ and -90 . The $-x$ eggs added to complete the culture were, however, not all of the same genotype but were half each from the remaining two genotypes of the experiment. These two will be referred to as the paired genotypes or components and that which 
provided the $120+x$ eggs as the unique genotype or component. Thus to take an example, the trioculture series in which $\mathrm{X} 1$ was used as the unique component and X5 and X15 as the paired components, the numerical composition of the mixtures of eggs used in setting up the cultures of this series were

\begin{tabular}{|c|c|c|c|c|c|}
\hline \multirow[b]{2}{*}{ Genotypes } & \multirow[b]{2}{*}{ Component } & \multirow[b]{2}{*}{ Composition } & \multicolumn{3}{|c|}{$x$} \\
\hline & & & -30 & -60 & -90 \\
\hline $\mathbf{X} 1$ & unique & $120+x$ & 90 & 60 & 30 \\
\hline$\times 5\}$ & & $-\frac{1}{2} x$ & 15 & 30 & 45 \\
\hline$X 15\}$ & paired & $-\frac{1}{2} x$ & 15 & 30 & 45 \\
\hline
\end{tabular}

When $\mathrm{X} 1$ is taken as the indicator the series is, of course, completed by the monocultures of $120 \mathrm{X} 1$ eggs, $x$ being 0 in the same sense as with duoculture analysis. The same amount of yeast was added as to the monoand duocultures, and this part of the experiment was done in duplicate in precise parallel with mono- and duoculture series and indeed was randomised with them. The trioculture series can thus be treated formally as a third series of duocultures and incorporated as such into the regression analysis with the unique genotype as indicator. Four regressions can then be calculated, all subject to the requirement for $p_{a}$ to have the same value, $e$, at the reference density of 120 indicator eggs per culture, viz., $b_{m}$ for the monoculture series, $b_{d 5}$ for the duocultures of X1 with X5, $b_{d 15}$ for the duocultures with $\mathrm{X} 15$, and $b_{d T}$ for the triocultures. The competition values, $c_{1,1}, c_{1,5}$ and $c_{1, T}$ an be derived from the regression coefficients in the customary way, the last three being $c_{1,5}=b_{d 5}-b_{m}$ and $c_{1,15}=b_{d 15}-b_{m}$ and $c_{1, T}=b_{d T}-b_{m}$.

The other two sets of triocultures, having X5 and X15 as the unique genotypes and now used as indicators, were set up and analysed in the same way. All twelve $c$ values obtained from the experiment are set out in table 1. Just as $c_{1 . T}$ is from the triocultures with $\mathrm{X} 1$ as the unique genotype, $c_{5 . T}$ is from the series with $\mathrm{X} 5$ as the unique and indicator genotype, and $c_{15, T}$ similarly from the series in which $\mathrm{X} 15$ played this part. The nine $c$ values derived from the mono- and duocultures are, of course, repeats of the entries in Mather and Caligari's (1983) table 9, which however did not include the $c$ 's from the triocultures.

TABLE 1

$c$ values from the $X$ experiment including those from the triocultures

\begin{tabular}{|c|c|c|c|c|}
\hline & Indicator & $\mathrm{X} 1$ & $\times 5$ & $\mathrm{X} 15$ \\
\hline \multirow{4}{*}{ 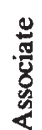 } & $\mathbf{X} 1$ & $0 \cdot 144003$ & 0.068583 & 0.017857 \\
\hline & $\times 5$ & 0.311035 & 0.295898 & 0.125725 \\
\hline & X15 & 0.180155 & 0.119749 & 0.085608 \\
\hline & TRIO & 0.306107 & 0.071047 & 0.119880 \\
\hline
\end{tabular}

\section{Competition in Triocultures}

Each of the three columns of table 1 includes the $c$ values representing the responses of the same indicator to competitive pressure from the other 
two genotypes used separately in duocultures and also used jointly as the paired genotypes in trioculture. The pressure exerted by the two associates when present together can thus be compared directly with the pressures exerted by these associates individually in the respective duocultures. Initially the triocultures were set up with equal numbers of eggs of the paired genotypes; but competition between the paired associates will have ensured that the numbers of individuals emerging finally as adult flies will not be equal since the $e$ and $r$ values of the genotypes were shown not to be equal by Mather and Caligari. The response of the common indicator to pressure from the paired associates must thus reflect the consequences of competition between them in the joint pressure they exert on the indicator.

Now to take the simplest hypothesis, if the joint pressure exerted.on the indicator by the paired associate genotypes in a trioculture is the sum of the pressures exerted by the individuals of the two genotypes we expect that, taking the case where $\mathrm{X} 1$ is the indicator, $c_{1, T}=u c_{1,5}+v c_{1,15}$ where $u$ and $v(=1-u)$ are the respective proportions that $\mathrm{X} 5$ and $\mathrm{X} 15$ contribute to the population of associate individuals over the period when competition is effective in the culture. We can thus test agreement of the observations with any expected values of $u$ and $v$ by testing whether $f=u c_{1,5}+v c_{1,15}-c_{1, T}$ departs significantly from 0 . This test requires that we know the value of $V_{f}$. Remembering that $c_{1,5}=b_{d 5}-b_{m}$ etc., and that all three $c$ values came from the same analysis of the set of data where X1 was the indicator, $b_{m}$ is the same for all of them. Thus

$$
f=u c_{1,5}+v c_{1,15}-c_{1, T}=u b_{d 5}+v b_{d 15}-b_{d T}
$$

The $J^{-1}$ matrix used in estimating the values of the regressions shows that $V_{b}$ will be the same for all $b$ at $V_{b}=37 V_{E} / 239,400$, and similarly all the covariances among the $b$ 's will be $W_{b, b}=18 V_{E} / 239,400$, where $V_{E}=$ 9.9095311 is the duplicate error variance. Thus $V_{f}=2(1-u v) \times$ $\left(V_{b}-W_{b, b}\right)=2(1-u v) 19 V_{E} / 239,400=0 \cdot 001572941(1-u v)$.

The value used here for $V_{E}$ requires comment as it is greater than that used earlier by Mather and Caligari (1983). Since the earlier analysis used only data from mono- and duocultures, the duplicate variance was derived from these two types of culture. In the present case the duplicate error variance from the triocultures was also brought into the pool, and it is greater than the mono- and duoculture values. A Bartlett test shows, however, that the estimates of $V_{E}$ from the three types of culture are not significantly heterogeneous $\left(\chi_{2}^{2}=4 \cdot 117, P=0 \cdot 13\right)$. It may be noted too that use of the present, greater value for $V_{E}$ does not alter the conclusions drawn from the earlier analysis of the mono- and duoculture data where the lower value of $V_{E}$ was used. The present value of $V_{E}$ is based on $72 \mathrm{df}$ as opposed to the $46 \mathrm{df}$ of the earlier estimate.

With the error variance of $f$ based on so many df, we can test the significance of its deviation from 0 by finding $\chi_{1}^{2}=f^{2} / V_{f}$. Using the ratio of eggs of the paired associates with which the triocultures were started, $u=v=\frac{1}{2}$ and $V_{f}=0.75 \times 0.001572941=0.00117971$ in all three cases. The results are set out in table 2 . The deviation of $f$ from 0 is getting close to significance in the comparison where $\mathrm{X} 1$ was the indicator; but taken overall the results show an acceptable fit with expectation $\chi_{3}^{2}=5 \cdot 517,(P=0 \cdot 14)$. Thus while the data fit the assumption of additivity of the selection pressures 
TABLE 2

Competitive pressure exerted on the unique genotype by the paired genotypes assuming $u=v=\frac{1}{2}$

\begin{tabular}{ccrrr}
\hline \multicolumn{2}{c}{ Genotypes } & & & \\
Unique & Paired & \multicolumn{1}{c}{$f$} & $\chi_{1}^{2}$ & $P$ \\
\hline X1 & X5, X15 & -0.060512 & 3.104 & 0.08 \\
X5 & X1,X15 & 0.023119 & 0.453 & 0.50 \\
X15 & X1, X5 & -0.048089 & 1.960 & 0.16 \\
\hline & & {$\left[\chi_{3}^{2}=\right] 5.517$} & 0.14 \\
\hline
\end{tabular}

In all cases $V_{f}=0 \cdot 00117971$.

exerted by the paired associates, there is no good evidence that the joint pressure of the associates on the indicator is affected, as would be expected, by the outcome of competition between the associates themselves.

Equally, however, instead of finding $u$ and $v$ from the numbers of eggs of the paired genotypes with which the triocultures were started, we can derive their values from the relative numbers of adults of the paired associates which eventually emerged, which must reflect the competitive interactions between these two genotypes. These relative numbers can of course be found from the triocultures themselves, but they can also be obtained from the duocultures involving the two genotypes which acted as joint associates in the trioculture, and the data from the relevant duocultures are more extensive than those from the triocultures. For example in the triocultures where X5 and X15 were the paired associates, X5 yielded a total of 113 adults but X15 only 65 . The X5, X15 duocultures similarly produced 344 adults of X5 and 214 of X15. Pooling the two sets of results, which are homogeneous, we find 457 adults of X5 and 279 of X15, giving $u=457 / 736=0 \cdot 621$ and $v=0 \cdot 379$. The values of $u$ and $v$ found in this way are set out in table 3 , together with those of $f, V_{f}$ and $\chi_{1}^{2}$ to which they lead. The near significant $\chi_{1}^{2}$ shown in table 2 by the triocultures with indicator $\mathrm{X} 1$ is now greatly reduced as is the second largest $\chi_{1}^{2}$, from the triocultures with indicator $\mathrm{X} 15$, and the $\chi_{1}^{2}$ from the remaining triocultures is only slightly increased. The overall fit is also materially improved $\left(\chi_{3}^{2}=\right.$ $2 \cdot 570, P=0.46)$. Thus although the fit based on $u=v=\frac{1}{2}$ was not so poor as to exclude this possibility, the fit based on the relative numbers of adults

TABLE 3

Competitive pressure exerted jointly by the paired genotypes when $u$ and $v$ are the relative proportions of adults recovered

\begin{tabular}{ccccrrrr}
\hline \multicolumn{2}{c}{ Genotypes } \\
Unique & Paired & $u$ & $v$ & $f$ & $V_{f}$ & $\chi_{1}^{2}$ & $P$ \\
\hline X1 & X5, X15 & 0.621 & 0.379 & -0.044676 & 0.00120274 & 1.659 & 0.20 \\
X5 & X1, X15 & 0.310 & 0.690 & 0.032841 & 0.00123649 & 0.872 & 0.35 \\
X15 & X1, X5 & 0.123 & 0.877 & -0.007423 & 0.00140327 & 0.039 & 0.84 \\
\hline \multicolumn{1}{r}{} \\
\hline
\end{tabular}


produced by the paired associates in triocultures and in corresponding duocultures is materially better. The results are not conclusive, but they clearly suggest that the competitive pressure exerted on the indicator in these triocultures is the sum of the pressures stemming from the individuals of the paired associate genotypes with their numbers as modified by the effects of competition they exerted on each other. This implies, too, that as might be expected the competition between the two associates went on at the same time as that by which they affected the indicator.

Continuing with our example of the trioculture series where the unique genotype was X1 and the paired genotypes X5 and X15, we can take the situation in reverse and enquire into the effect of competitive pressure from $\mathrm{X} 1$ on the expression of character $p_{a}$ in X5 and X15. Unfortunately this cannot be examined by the same kind of regression anaysis that we used above, because the experiment did not include the equivalent of a monoculture series for X5 and X15 taken together, i.e., a series of cultures all of which were set up with equal numbers of eggs from X 5 and X15 but with the total densities of eggs varying in the way characteristic of monoculture series. This is a deficiency that could be remedied in later experiments, but in its absence we must adopt a different approach.

Competitive pressure is exerted, at least prospectively, by all the individuals of a group or population on every one of their number, and so is a property of the group as a whole. Thus given that the contributions of the various genotypes in the group are simply additive, as we have been led to postulate, we expect that the average pressure exerted on each and every individual in this group will be $u_{1} a_{1}+u_{2} a_{2} \cdots=S(u a)$, where $u_{1}, u_{2}, \ldots$ are the proportions that genotypes $1,2, \ldots$ constitute of the group, and $a_{1}, a_{2}, \ldots$ are their $a$ (aggression) values. So with a duoculture of density 120 , containing equal numbers of X5 and X15 individuals, the average pressure exerted on any individual in the group will be $\frac{1}{2}\left(a_{5}+a_{15}\right)$, irrespective of whether that individual is X5 or X15. With a trioculture of the same density but now having 60 individuals of $X 1$ in addition to 30 each of X5 and X15, the average pressure exerted will be $\frac{1}{4}\left(2 a_{1}+a_{5}+a_{15}\right)$, and again this pressure will be exerted on all individuals whether X5 or $\mathrm{X} 15$, and indeed on $\mathrm{X} 1$ too.

Now, while the pressure of competition exerted on an individual is a property of the group, the degree of response to that pressure is not a property of the group but of the individual itself. For an X5 individual it will be $r_{5}$ and for an X15 it will be $r_{15}$. Since there is no evidence that the $a$ and $r$ components are other than additive in this experiment, we can thus write the $c$ values of $\mathrm{X} 5$ and $\mathrm{X} 15$ in duo- and triocultures of the composition we are discussing as shown in table 4 . If now we find the sums and differences of the values for X5 and X15 in each type of culture separately, we find that the difference is the same in duo- and triocultures but the sum is not, as is also shown in the table. Subtracting the trioculture sum from that of the duocultures their difference is $\frac{1}{2}\left(a_{5}+a_{15}-2 a_{1}\right)$. Thus where $a_{1}>$ $\frac{1}{2}\left(a_{5}+a_{15}\right), c$ will be higher in the trioculture than the duoculture; and since the higher the value of $c$ (the competitive value) the lower the value of $p_{a}$ (the expression of the character), $p_{a}$ will be lower in the trio- than duoculture. With $a_{1}<\frac{1}{2}\left(a_{5}+a_{15}\right), c$ will be lower and $p_{a}$ higher in trio- than in duoculture, and with $a_{1}$ intermediate between $a_{5}$ and $a_{15}$ the changes in both $c$ and $p_{a}$ are likely to be small. 
TABLE 4

$c$ values expected from paired genotypes in duoculture and trioculture

\begin{tabular}{ccc}
\hline Culture & X5 & Paired genotypes \\
\hline DUO & $\frac{1}{2}\left(a_{5}+a_{15}\right)+r_{5}$ & $\frac{1}{2}\left(a_{5}+a_{15}\right)+r_{15}$ \\
TRIO & $\frac{1}{4}\left(2 a_{1}+a_{5}+a_{15}\right)+r_{5}$ & $\frac{1}{4}\left(2 a_{1}+a_{5}+a_{15}\right)+r_{15}$ \\
\hline & Sum & Diff. \\
& $X 5+\mathrm{X} 15$ & $X 5-\mathrm{X} 15$ \\
\hline DUO & $\left(a_{5}+a_{15}\right)+\left(r_{5}+r_{15}\right)$ & $\left(r_{5}-r_{15}\right)$ \\
TRIO & $\frac{1}{2}\left(2 a_{1}+a_{5}+a_{15}\right)+\left(r_{5}+r_{15}\right)$ & $\left(r_{5}-r_{15}\right)$ \\
\hline
\end{tabular}

We have the relevant values of $p_{a}$ for $\mathrm{X} 5$ and $\mathrm{X} 15$ from both duoculture and trioculture at $x=-60$, but overall numbers of eggs on which they are based are not large, especially in the triocultures. They can, however, be effectively increased. Since the value of $p_{a}$ is linearly related to density, the average of the values shown by a genotype at $x=-30,-60$ and -90 will give an estimate of $p_{a}$ at $x=-60$, and this estimate will be based on nearly three times the information available from the value at $x=-60$ taken alone. This is true for both trioculture and duoculture, though of course $x$ must be read in opposite directions for the two genotypes in the case of duocultures. The values of $p_{a}$ so obtained are set out in table 5, for X5 and X15 in

TABLE 5

Values of $p_{a}$ shown by paired genotypes in duoculture $(D)$ and trioculture $(T)$

\begin{tabular}{|c|c|c|c|c|c|c|c|c|c|}
\hline & X5 & $\mathrm{X} 15$ & Sum & Diff. & & $\mathrm{X} 1$ & $\mathrm{X} 15$ & Sum & Diff. \\
\hline D1 & $31 \cdot 205$ & $22 \cdot 905$ & $54 \cdot 110$ & $8 \cdot 300$ & D1 & 17.430 & $26 \cdot 160$ & $43 \cdot 590$ & -8.730 \\
\hline D2 & $29 \cdot 385$ & $20 \cdot 685$ & 50.070 & 8.700 & D2 & 17.085 & $29 \cdot 743$ & $46 \cdot 828$ & -12.658 \\
\hline $\mathrm{T}$ & 35.468 & $25 \cdot 282$ & $60 \cdot 750$ & $10 \cdot 186$ & $\mathrm{~T}$ & 12.678 & 22.532 & $35 \cdot 210$ & -9.854 \\
\hline
\end{tabular}

\begin{tabular}{crccc}
\hline & \multicolumn{1}{c}{ X1 } & X5 & Sum & Diff. \\
\hline D1 & 9.928 & 33.500 & 43.428 & -23.572 \\
D2 & 5.233 & 32.675 & 37.908 & -27.442 \\
T & 12.700 & 32.575 & 42.275 & -19.875 \\
\hline
\end{tabular}

(X15 added in T)

both their duoculture series and the trioculture in which they are the paired genotypes with $\mathrm{X} 1$ as the unique one. The values of $p_{a}$ in the other two combinations, $\mathrm{X} 1$ with $\mathrm{X} 5$, and $\mathrm{X} 1$ with $\mathrm{X} 15$, are also set out in the table. Two complete sets of duocultures were available, though only one of triocultures. The results from the two sets of duocultures are denoted as D1 and D2, and are given separately in the table. They serve to provide a test of consistency of the experimental findings. 
Since $p_{a}$, the proportion of eggs successfully developing into adult flies, has been transformed into angles, its variance is independent of the value of $p_{a}$ itself, and depends only on the number of eggs used in finding $p_{a}$. As we have seen each entry in table 5 is the mean of three values of $p_{a}$, one each from cultures where $x=-30,-60$ and -90 respectively. Of the 360 eggs involved in finding each value of $p_{a}$ from duocultures, 180, 120 and 60 came respectively from cultures at these three values of $x$. Since in general $V_{p a}=820 \cdot 7 / n$, where $n$ is the number of eggs, the mean of three values will have

$$
V_{p a D}=\frac{820 \cdot 7}{9}\left(\frac{1}{180}+\frac{1}{120}+\frac{1}{60}\right)=2 \cdot 786327
$$

The numbers of eggs used in triocultures at corresponding values of $x$, are shared equally between the two relevant genotypes, and so we find

$$
V_{p a T}=2 V_{p a D}=5 \cdot 572654 \text {. }
$$

Returning to the observations in table 5, since we have two values of $p_{a}$ for duocultures (D1 and D2) and one for triocultures (T), there are two degrees of freedom among the three sums and two among the three differences. Taking the paired genotypes X5 and X15 as an example and writing $p_{a 5 D 1}, p_{a 5 D 2}$ and $p_{a 5 T}$ for the three values from X5 and similarly for $\mathrm{X} 15$, the three sums from $\mathrm{D} 1, \mathrm{D} 2$ and $\mathrm{T}$ are $p_{a 5 D 1}+p_{a 15 D 1}, p_{a 5 D 2}+p_{a 15 D 2}$, and $p_{a 5 T}+p_{a 15 T}$ among which we can make the two relevant comparisons

$$
\begin{gathered}
\frac{1}{4}\left[\left(p_{a 5 D 1}+p_{a 15 D 1}\right)-\left(p_{a 5 D 2}+p_{a 15 D 2}\right)\right] \text { and } \\
\frac{1}{12}\left[\left(p_{a 5 D 1}+p_{a 15 D 1}\right)+\left(p_{a 5 D 2}+p_{a 15 D 2}\right)-2\left(p_{a 5 T}+p_{a 15 T}\right)\right] .
\end{gathered}
$$

The former tests for consistency of the sum over the two groups of duocultures, which we expect, and the latter tests for constancy between duo- and triocultures which we expect not to find in all cases. The corresponding S.S. are of course

$$
\begin{gathered}
\frac{1}{4}\left[\left(p_{a 5 D 1}+p_{a 15 D 1}\right)-\left(p_{a 5 D 2}+p_{a 15 D 2}\right)\right]^{2} \text { and } \\
\frac{1}{12}\left[\left(p_{a 5 D 1}+p_{a 15 D 1}\right)+\left(p_{a 5 D 2}+p_{a 15 D 2}\right)-2\left(p_{a 5 T}+p_{a 15 T}\right)\right]^{2}
\end{gathered}
$$

which, on substituting the values from the table become

$$
\begin{gathered}
\frac{1}{4}(54 \cdot 110-50 \cdot 070)^{2}=4 \cdot 0804 \text { and } \\
\frac{1}{12}(54 \cdot 110+50 \cdot 070-2 \times 60 \cdot 750)^{2}=24.9985
\end{gathered}
$$

respectively.

Turning from the sums of $p_{a 5}$ and $p_{a 15}$ to their difference, we now expect to find no significant difference, not only between D1 and D2 but also between D1 and D2 on the one hand and T on the other. The relevant comparisons and the S.S. they account for can be obtained by substituting $\left(p_{a 5 D 1}-p_{a 15 D 1}\right)$ for $\left(p_{a 5 D 1}+p_{a 15 D 1}\right)$ and so on in the above expression. Again substituting the observed values from table 5 we find the two S.S. to be $\frac{1}{4}(8 \cdot 300-8 \cdot 700)^{2}=0 \cdot 0400$ and $\frac{1}{12}(8 \cdot 300+8 \cdot 700-20 \cdot 372)^{2}=0 \cdot 9475$. These results and the corresponding S.S. for the other pairs of genotypes are set out in table 6 .

The significance of these comparisons, each corresponding to $1 \mathrm{df}$, can be tested against the sampling variances of the estimates of $p_{a}$, as found earlier. Each value of $p_{a}$ has a sampling variance $V_{p a D}$, and each from a 
TABLE 6

Analysis of $p_{a}$ values in Table 5

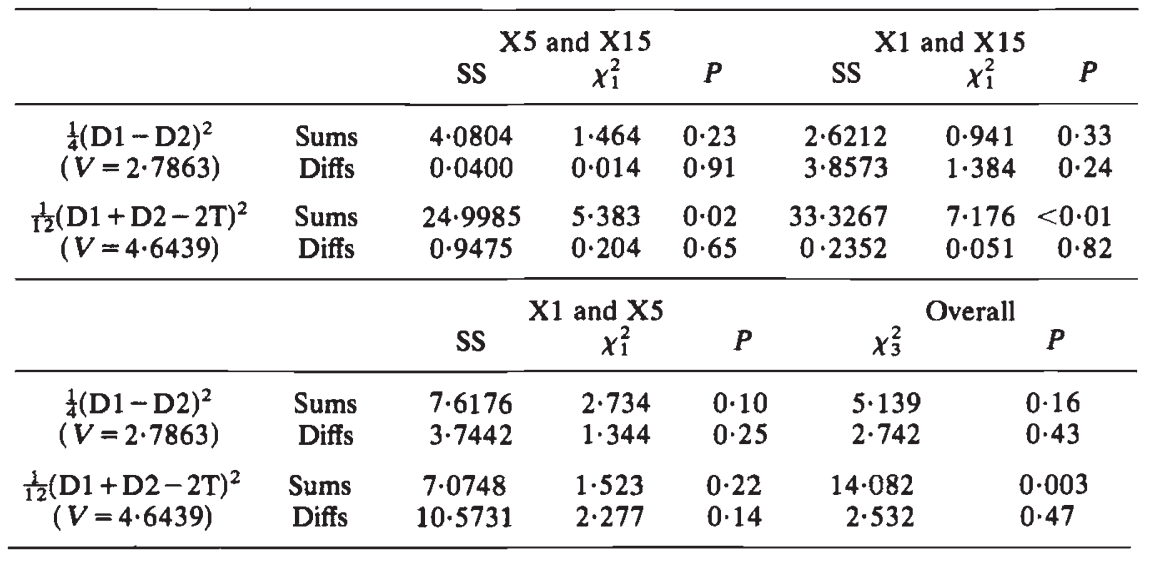

trioculture a variance of $V_{p a T}=2 V_{p a D}$. Then each comparison of D1 with $\mathrm{D} 2$, which involves four values of $p_{a}$ from duocultures, the sampling variance will be $\frac{1}{4}\left(4 V_{p a D}\right)=V_{p a D}=2 \cdot 7863$; and each comparison of D1 + D2 with $2 \mathrm{~T}$ will similarly have a sampling variance of $\frac{1}{12}\left(4 V_{p a D}+8 V_{p a T}\right)=4 \cdot 6439$. Dividing the S.S. by the appropriate sampling variances yields the $\chi_{1}^{2} \mathrm{~s}$ as set out, together with their probabilities, in table 6 .

There is no significant difference between D1 and D2 in respect of either sums or differences: and if we pool the three $\chi_{1}^{2}$ from the three pairs of genotypes we find $\chi_{3}^{2}$ which again shows no significance. In other words, the results from D1 and D2 are consistent with one another as expected. The comparisons between duoculture and trioculture similarly show no significance in respect of the differences, whether taken individually from each of the three pairs of genotypes or pooled to give an $\chi_{3}^{2}$, again as expected. But the comparisons, D1 $+\mathrm{D} 2-2 \mathrm{~T}$, between the combined duocultures and the triocultures, are highly significant for the pairs $\mathrm{X} 1$ with X15 $(P<0.01)$ and X5 with X15 $(P=0.02)$, though not significant for the pair X1 with X5 $(P=0 \cdot 22)$. Pooling the three $\chi_{1}^{2}$, however, gives $\chi_{3}^{2}=14.082$ with $P=0.003$, and there can be no doubt that although the differences show no evidence of changing from duo- to triocultures, the sums do so-in both cases as expected.

We can take the test of agreement with expectation a step further. Mather and Caligari (1983) showed that of the three genotypes X1 is the weakest aggressor, $\mathrm{X} 5$ is the strongest and $\mathrm{X} 15$ is intermediate. We expect therefore that the introduction of X1 into the mixture of X5 and X15 would reduce the competitive pressure on both of this pair of genotypes, and that their $p_{a}$ would thus rise in the trioculture by comparison with the duoculture, as reference to table 5 shows that indeed it does. Similarly the introduction of $\mathrm{X} 5$ into the mixture of $\mathrm{X} 1$ and $\mathrm{X} 15$ should increase the competitive pressure and lower the $p_{a}$ of both of them in the trioculture, again as expected. Finally the introduction of X15 into the mixture of X1 and X15 should have a relatively small effect on the competitive pressure and hence of the $p_{a}$ of these two, and this is the only case where there is no evidence of a difference between duocultures and triocultures. 


\section{Conclusion}

When the unique genotype in a trioculture was used as the indicator and the competitive pressure exerted jointly by the paired genotypes was compared with the pressures exerted by these two genotypes individually in duoculture with the same indicator, the joint pressure by the paired genotypes was intermediate between those of its two individual constituents. Furthermore, although the joint pressure did not depart from the simple mean of those of the two constituents, it fitted much better with the mean found by weighting according to the relative proportions of the two genotypes among the individuals recovered from both the triocultures and the duocultures between them, thus clearly suggesting that the effects of competition between the paired genotypes was reflected, as one must expect it to be, in the pressure they jointly exerted. In other words, these results pointed to the joint pressure being $a_{j}=u a_{1}+v a_{2}$, where $a_{j}$ is the joint pressure, $a_{1}$ and $a_{2}$ those of the individual constituents and $u$ and $v(=1-u)$ the proportions of the two constituents at the end of the competitive processes. In this sense there is ground for inferring that the competitive pressures exerted by different genotypes are simply additive.

A similar analysis could not be undertaken in the opposite direction, taking the unique genotype of the trioculture as an associate and the paired genotypes as indicators, because the experiment did not include a series using the paired genotypes in a way corresponding to a monoculture series with a single genotype. Nevertheless, comparisons made between the values of $p_{a}$ shown by the paired genotypes at $x=-60$ in the trioculture and at $x=-60$ in simple duocultures between them, yielded results which agreed well with expectations based on this simple additive relation. Obviously, more evidence from larger experiments, which include the paired genotypes' equivalent of monocultures, which utilise initial ratios of eggs other than $u=v=\frac{1}{2}$, and which extend to more complex mixtures of genotypes, say quarto- and even quintocultures, will be needed before we can be confident of this conclusion. The approach which has proved fruitful with the present data, and which can be extended to more complex relations, provides a basis for both designing and analysing such extended experiments. And given that the additive relation holds good, it would provide a basis both for assessing the effects of competition in a complex population and for a biometrico-genetical analysis of the determination of these effects.

Acknowledgements. We gratefully acknowledge financial support from the Agricultural Research Council. We are also grateful to Dr C. Haley who carried out the Bartlett test and computed the $\chi^{2}$ probabilities where $\chi^{2}$ had more than $1 \mathrm{df}$.

\section{REFERENCES}

CALIGARI, P. D. S. 1980.Competitive interactions in Drosophila melanogaster. I. Monocultures. Heredity, 45, 219-231.

MATHER, K AND CALIGaRI, P. D. S. 1981. Competitive interactions in Drosophila melanogaster. II. Measurement of competition. Heredity, 46, 239-254.

MATHER, K. AND CALIGARI, P. D. S. 1983. Pressure and response in competitive interactions. Heredity, 51, 435-454.

MATHER, K., HILL, J. AND CALIGARI, P. D. S. 1983. Analysis of competitive ability among genotypes of perennial ryegrass. Heredity, 48, 421-434. 